\section{Case Reports in Oncology}

\title{
Occurrence of an Abscopal Radiation Recall Phenomenon in a Glioblastoma Patient Treated with Nivolumab and Re-Irradiation
}

\author{
Wouter O. van Seggelen ${ }^{a} \quad$ Filip Y. De $\operatorname{Vos}^{c}$ Heike Röckmann ${ }^{a}$ \\ Marijke R. van Dijk ${ }^{d} \quad$ Joost J.C. Verhoeff ${ }^{b}$ \\ aDepartment of Dermatology and Venerology, University Medical Center Utrecht, \\ Utrecht, The Netherlands; ${ }^{\text {b}}$ Department of Radiation Oncology, University Medical Center \\ Utrecht, Utrecht, The Netherlands; 'Department of Medical Oncology, University Medical \\ Center Utrecht, Utrecht, The Netherlands; ${ }^{d}$ Department of Pathology, University Medical \\ Center Utrecht, Utrecht, The Netherlands
}

\section{Keywords}

Abscopal effect $\cdot$ Radiation recall $\cdot$ Nivolumab $\cdot$ Glioblastoma

\begin{abstract}
Glioblastoma multiforme is the most frequent primary brain tumor. The clinical course of glioblastoma is almost invariably fatal. Combined chemo-irradiation with temozolomide is currently the standard of care for newly diagnosed glioblastoma and concurrent Nivolumab, an anti-PD-1 monoclonal antibody is being studied for de novo glioblastoma. We present a 62year old patient with glioblastoma, which was discovered during evaluation of sudden-onset moderate ataxia. Following craniotomy of the glial tumour he received chemo radiation. During this first-line treatment the patient participated in the CA209-548 phase III placebo controlled study investigating the addition of concurrent nivolumab. One month after the last administration of nivolumab after 60 weeks of study participation, magnetic resonance imaging scan showed progressive disease. Therefore stereotactic re-irradiation was given. Five days after completing radiation therapy and 50 days after his last nivolumab course he developed a mild diffuse generalized pruritic maculopapular exanthema. Skin biopsy was very indicative for a drug hypersensitivity reaction. The maculopapular rash and pruritus was successfully
\end{abstract}


treated with moderate potency topical corticosteroids and prednisone. With the introduction of PD1/PD-L1 inhibitors and other immunotherapies tweaking the immune system to target cancer cells one can argue that once local radiation triggers a local immune mediated hypersensitivity reaction as seen in radiation recall dermatitis, the subsequent hypersensitivity reaction which would traditionally only be a local reaction is now possible to advance to more pronounced (systemic) reactions as seen in an abscopal effect. Therefore, we propose a combined name to coin this effect, the abscopal radiation recall phenomenon.

(C) 2019 The Author(s)

Published by S. Karger AG, Basel

\section{Introduction}

Glioblastoma multiforme (GBM) is the most frequent primary brain tumor and is of astrocytic origin. The clinical course of GBM is almost invariably fatal, with a median survival of 12 months [1]. The addition of temozolomide (TMZ) to 60 Gy of radiotherapy improves two-year survival from $11 \%$ to $27 \%$, and combined chemo-irradiation with TMZ is currently the standard of care for newly diagnosed GBM [2]. Once a GBM recurs however, the treatment options are limited. The mechanistic rationale supporting cancer immunotherapy rests on the premise that tumors can be recognized as foreign rather than as self and can be effectively attacked by an activated immune system [3].

The anti-PD-1 monoclonal antibody nivolumab is a fully-human monoclonal immunoglobulin (Ig) G4 antibody which binds to the PD-1 cell surface membrane receptor expressed by activated $\mathrm{T}$ and $\mathrm{B}$ lymphocytes, blocking the interaction between PD 1 and its ligands and down-regulating antigen-specific $\mathrm{T}$ cell responses. Nivolumab is currently being studied in two phase 3 studies with de novo glioblastoma (NCT02617589 and NCT02667587) [4].

\section{Case Presentation}

A 62-year old man underwent a craniotomy with total resection of a glial tumour in the right sided fronto-parietal brain lobe, which was discovered during evaluation of sudden-onset moderate ataxia. Histological examination revealed IDH (isocitrate dehydrogenase) wild type glioblastoma multiforme with MGMT (0-6-Methylguanine-DNA Methyltransferase) hypermethylation. Post-operative chemoradiation was given with a total dose of 30 fractions of 2 Gy and temozolomide $75 \mathrm{mg} / \mathrm{m}^{2} /$ day followed by six courses of temozolomide $200 \mathrm{mg} / \mathrm{m}^{2}$ days 1-5 every 4 weeks. During this first-line treatment the patient participated in the CA209548 phase III placebo controlled study investigating the addition of nivolumab with standard chemoradiation. From August 2017 till August 2018 the patient received 19 courses of nivolumab/placebo of which the first eight courses were given every 2 weeks at fixed dose of $240 \mathrm{mg}$ and following courses every 4 weeks at fixed dose of $480 \mathrm{mg}$ according to study protocol. One month after the last administration of nivolumab/placebo, magnetic resonance imaging (MRI) scan showed progressive disease after 60 weeks of study participation. At that time, the patient had some minor complaints not related to treatment: cognitive disorder grade 1 , depth perception grade 1 , neuropathy grade 1 and fatigue grade 1 . Per advice of the multidisciplinary tumour board, a FET-PET-scan was performed, confirming a compact area of recurrent disease at the border of the radiation field. Subsequently, stereotactic re-irradiation was given with three fractions of $7 \mathrm{~Gy}$ on the lesion in the right periventricular region. No prophylactic dexamethasone was administered as no risk of radiation-induced edema was 
expected. Five days after completing radiation therapy and 50 days (1.6 months) after his last nivolumab/placebo course and 15.6 months after initiating the study he developed a maculopapular rash in both armpits (Fig. 1).

The patient (Fitzpatrick skin type 1) was evaluated by the dermatology department and presented with a mild diffuse generalized pruritic maculopapular exanthema with excoriations starting in axillar region and later more pronounced on the ventral side of the arms and proximal area of the legs. The skin of the head region was not involved.

Skin biopsy revealed a minimally spongiotic epidermis with slight exocytosis of lymphocytes in the epidermis. In the superficial dermis small blood vessels with a lympho-histiocytic infiltrate including interstitial eosinophilic granulocytes were present. The histology was very indicative for a drug hypersensitivity reaction.

The maculopapular rash was treated with moderate potency topical corticosteroids and prednisone $40 \mathrm{mg}$ once daily for a total duration of 12 days in a tapered schema. The pruritus and rash resolved within 6 weeks.

Because of suspected (systemic) radiation recall phenomenon we obtained permission from the study sponsor, BMS, for unblinding procedure revealing he was treated in the nivolumab arm. Careful evaluation of all drug exposures revealed that besides metformin, enalapril and levetiracetam; nivolumab was the only additional drug given in the past 40 weeks.

\section{Discussion and Conclusion}

Nivolumab is an anti-PD-1 monoclonal antibody a checkpoint inhibitor also known as an immunomodulator; activating the patient's immune system to tackle cancer cells [5]. By stimulating the patient's immune system this can result in immune system related adverse events, most commonly the gastrointestinal tract, endocrine glands, skin, and liver [6]. Cutaneous adverse events occur in $40 \%$ of patients who receive nivolumab, most commonly maculopapular rashes (15-21.7\%) and pruritus (17-18.8\%). Less frequently vitiligo, psoriasis and mucosal complaints are reported [7]. Generally, cutaneous adverse events occur 4.2 months (median; range of 0.5-38.0 months) after starting nivolumab. Cutaneous adverse reactions have also developed after the drug therapy was terminated [8]. Interestingly, in our patient, his generalized cutaneous adverse event was closely associated with the re-initiation of local radiotherapy, reminding us of the abscopal effect.

In 1953, Dr. R.H. Mole first coined this term as "an action at a distance from the irradiated volume but within the same organism" [9]. This effect is still poorly understood, it is postulated that local radiation can trigger complex tissue responses that can have systemic effects on tumor growth and/or inhibition [10]. In our patient the effect on tumor growth was inconclusive as subsequent MRI showed some degree of tumor progression; however this could also have been radionecrosis, a result of tissue necrosis due to high dose (re-) irradiation. The patient deceased 4 months later.

The strong correlation between radiation and the notable generalized cutaneous reaction shortly thereafter suggests that the localized radiation initiated an immune mediated response. A similar effect can be observed in patients who receive anti-cancer agents and subsequent radiation [11], as was the case in our patient. This phenomenon is widely recognized as radiation recall dermatitis (RRD), however for RRD, the location of the dermatitis is completely shaped like the projected radiotherapy field that was administered earlier. This phenomenon has been acknowledged after the introduction of the first cytotoxic therapy over 60 years ago and has been described for the majority of the drugs commonly used in cancer 
therapy [12]. The underlying mechanism is poorly understood; it is proposed that it is a hypersensitivity reaction, induced by administration of chemotherapy and recalled by subsequent radiation. The occurrence of RRD can range from a few days up to years later.

With the introduction of PD1/PD-L1 inhibitors and other immunotherapies tweaking the immune system to target cancer cells, one can argue that once local radiation triggers a local immune mediated hypersensitivity reaction as seen in RRD, the subsequent hypersensitivity reaction which would traditionally only be a local reaction is now enabled to advance to more pronounced (systemic) reactions as seen in abscopal effects due to the immunomodulatory effects of the immunotherapy. Therefore, we propose a combined name to coin this effect, the abscopal radiation recall phenomenon (ARRP). This phenomenon could theoretically have clinical implications for future treatment models as it could be used as a marker for treatment response and/or be an adverse effect which needs to be recognized and treated effectively. We present, to our knowledge, the first case of an abscopal radiation recall phenomenon (ARRP).

\section{Statement of Ethics}

Patient gave informed consent to publish their case (including publication of images).

\section{Disclosure Statement}

The authors have no conflicts of interest to declare.

\section{Funding Sources}

There was no funding provided for the writing of this manuscript.

\section{Author Contributions}

All authors contributed to the writing and review of the manuscript.

\section{References}

1 DeAngelis LM. Brain tumors. N Engl J Med. 2001 Jan;344(2):114-23.

2 Stupp R, Mason WP, van den Bent MJ, Weller M, Fisher B, Taphoorn MJ, et al.; European Organisation for Research and Treatment of Cancer Brain Tumor and Radiotherapy Groups; National Cancer Institute of Canada Clinical Trials Group. Radiotherapy plus concomitant and adjuvant temozolomide for glioblastoma. N Engl J Med. 2005 Mar;352(10):987-96.

3 Dunn GP, Old LJ, Schreiber RD. The immunobiology of cancer immunosurveillance and immunoediting. Immunity. 2004 Aug;21(2):137-48.

4 Postow MA, Sidlow R, Hellmann MD. Immune-Related Adverse Events Associated with Immune Checkpoint Blockade. N Engl J Med. 2018 Jan;378(2):158-68.

5 Ito A, Kondo S, Tada K, Kitano S. Clinical Development of Immune Checkpoint Inhibitors. BioMed Res Int. 2015;2015:605478.

6 Weber JS, Hodi FS, Wolchok JD, Topalian SL, Schadendorf D, Larkin J, et al. Safety Profile of Nivolumab Monotherapy: A Pooled Analysis of Patients With Advanced Melanoma. J Clin Oncol. 2017 Mar;35(7):78592. 


\section{Case Reports in Oncology}

7 Sibaud V, Meyer N, Lamant L, Vigarios E, Mazieres J, Delord JP. Dermatologic complications of anti-PD-1/PDL1 immune checkpoint antibodies. Curr Opin Oncol. 2016 Jul;28(4):254-63.

8 Wang LL, Patel G, Chiesa-Fuxench ZC, McGettigan S, Schuchter L, Mitchell TC, et al. Timing of Onset of Adverse Cutaneous Reactions Associated With Programmed Cell Death Protein 1 Inhibitor Therapy. JAMA Dermatol. 2018 Sep;154(9):1057-61.

9 Mole RH. Whole body irradiation; radiobiology or medicine? Br J Radiol. 1953 May;26(305):234-41.

10 Formenti SC, Demaria S. Systemic effects of local radiotherapy. Lancet Oncol. 2009 Jul;10(7):718-26.

11 Hird AE, Wilson J, Symons S, Sinclair E, Davis M, Chow E. Radiation recall dermatitis: case report and review of the literature. Curr Oncol. 2008 Jan;15(1):53-62.

12 Azria D, Magné N, Zouhair A, Castadot P, Culine S, Ychou M, et al. Radiation recall: a well recognized but neglected phenomenon. Cancer Treat Rev. 2005 Nov;31(7):555-70.

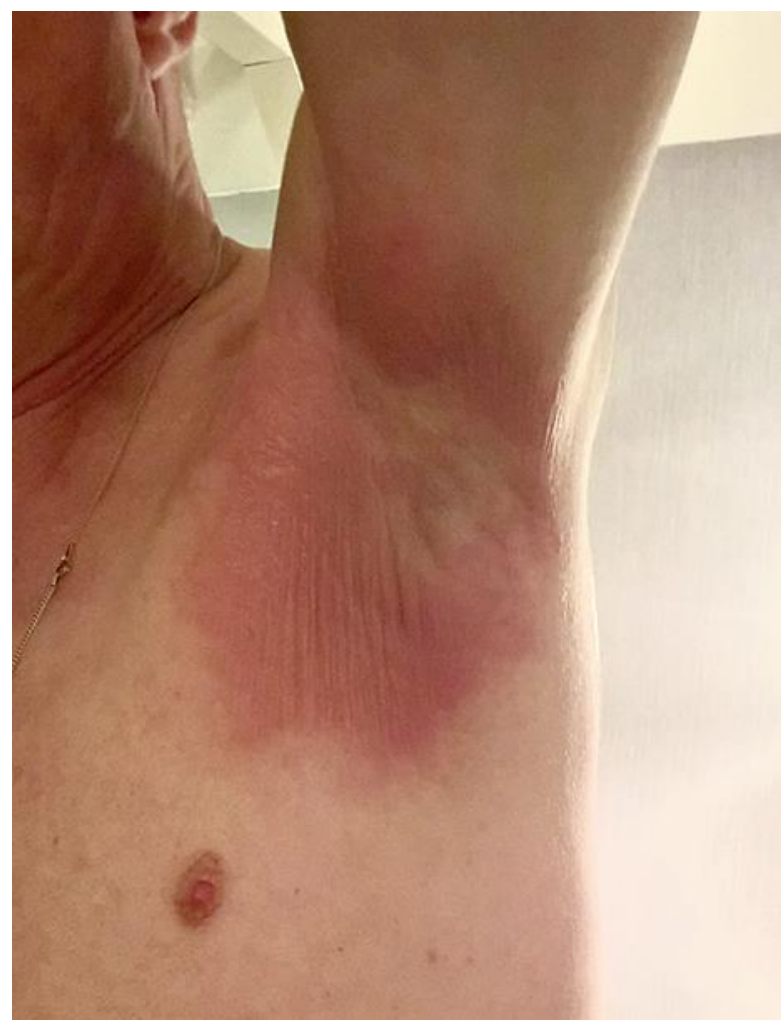

Fig. 1. Characteristic maculopapular rash, initially in the axillae and later more diffuse all over the patient's body. 